Supporting Information

\title{
Electrowetting Behavior and Digital Microfluidic Applications of Fluorescent, Polymer-Encapsulated Quantum Dot Nanofluids
}

Urice N. Tohgha, ${ }^{\dagger, \S}$ Ernest L. Alvino, ${ }^{\ddagger}$ Clark C. Jarnagin, ${ }^{\dagger, \S}$ Scott T. Iacono, ${ }^{\ddagger}$ Nicholas P. Godman ${ }^{*} \dagger$

†Air Force Research Laboratory, Materials and Manufacturing Directorate, Wright-Patterson Air Force Base, Dayton, Ohio 45433-7750, United States

§Azimuth Corporation, 4027 Colonel Glenn Highway, Beavercreek, Ohio 45431, United States

$\ddagger$ Department of Chemistry and Chemistry Research Center, United States Air Force Academy, Colorado Springs, Colorado 80840, United States

Correspondence e-mail: nicholas.godman.2@us.af.mil 


\section{Contents}

Figure S1. TEM images of QD-G-COOH, QD-G-PDDA and QD-G-PEG.

Figure S2. Normalized absorption and emission spectra of QD-G-COOH, QD-G-PDDA and QD-G-PEG. .S-4

Table S1. Contact angle and interfacial tension values of all nanofluids in addition to PAA and $\mathrm{DI} \mathrm{H}_{2} \mathrm{O}$ in dodecane oil.

Figure S3. Representative sessile droplet images of QD-G-COOH, QD-G-PDDA, and QD-G-PEG depicting contact angle hysteresis.

Figure S4. TEM images of QD-G-COOH at two dilutions depicting the effect of excess polymer.

Figure S5. Contact angle change as a function of applied voltage for various concentrations of QD-G$\mathrm{COOH}$

Figure S6. Normalized absorption and emission spectra of QD-G-COOH, QD-Y-COOH, QD-O-COOH, and QD-R-COOH.

Figure S7. TEM images of QD-G-COOH, QD-Y-COOH, QD-O-COOH, and QD-R-COOH S-10

Table S2. Contact angle and interfacial tension values of nanofluids in $0.05 \mathrm{wt} \%$ and $1.0 \mathrm{wt} \%$ span 80 in dodecane oil. 

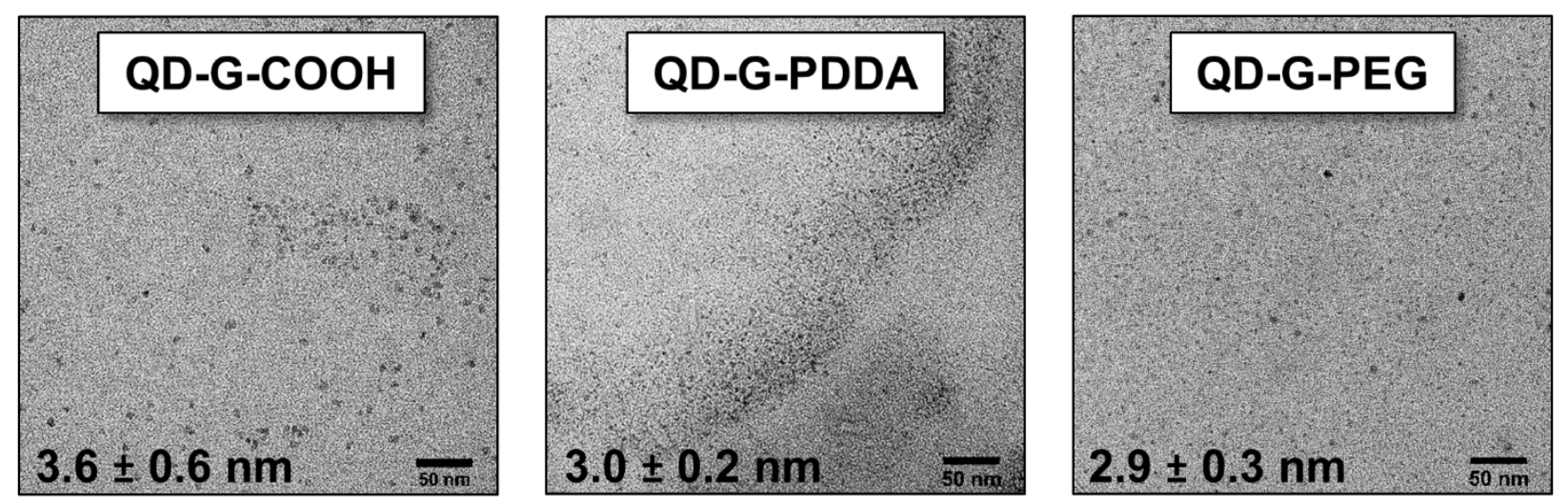

Figure S1. TEM images of QD-G-COOH, QD-G-PDDA and QD-G-PEG. Samples were diluted 40 times with DI water. 

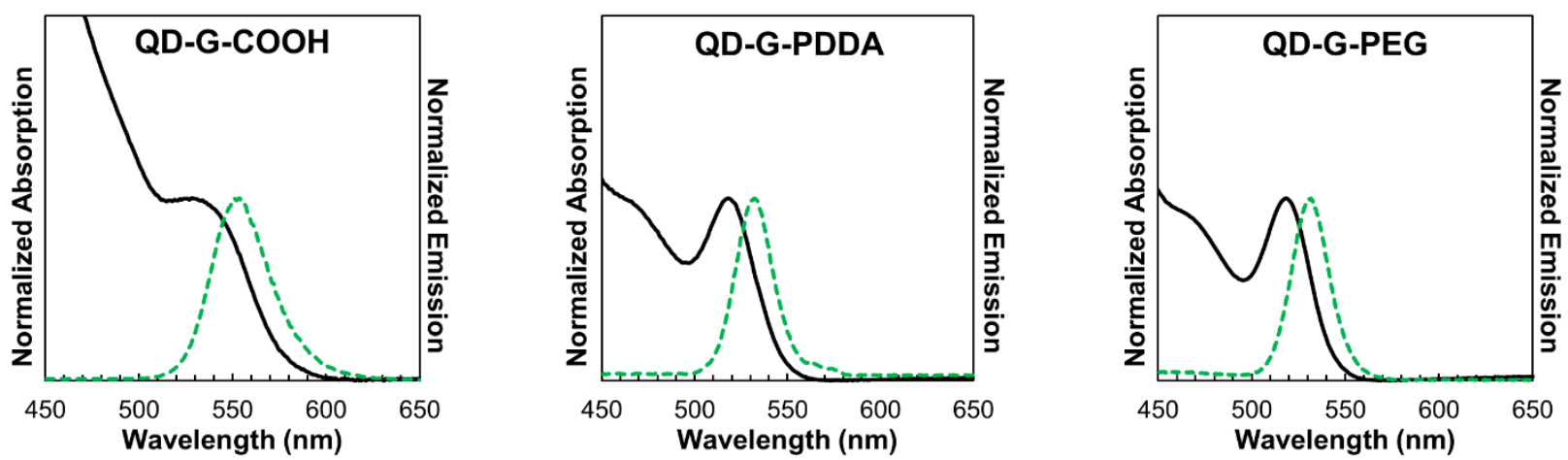

Figure S2. Normalized absorption (black) and emission (green) spectra of QD-G-COOH, QDG-PDDA and QD-G-PEG. 


\begin{tabular}{|l|l|l|l|l|l|l|}
\cline { 2 - 7 } \multicolumn{1}{c|}{} & $\theta_{0 \mathrm{~V}}\left(^{\circ}\right)$ & $\theta_{\mathrm{sat}}\left(^{\circ}\right)$ & $\Delta \theta_{5 \mathrm{~V}}\left(^{\circ}\right)$ & $\Delta \theta_{\text {sat }}\left(^{\circ}\right)$ & $\begin{array}{l}\gamma_{L L} \\
(\mathrm{mN} / \mathrm{m})\end{array}$ & $\begin{array}{l}\gamma_{L L, a p p} \\
(\mathrm{mN} / \mathrm{m})\end{array}$ \\
\hline QD-G-PDDA & $159.7 \pm 1.7$ & $68.6 \pm 1.8$ & $19.7 \pm 3.1$ & $91.1 \pm 0.7$ & $31.2 \pm 0.7$ & $11.9 \pm 0.7$ \\
QD-G-PEG & $158.4 \pm 1.5$ & $59.5 \pm 0.8$ & $18.1 \pm 1.5$ & $98.9 \pm 0.8$ & $30.8 \pm 0.2$ & $12.8 \pm 0.3$ \\
Water & $162.2 \pm 1.0$ & $79.4 \pm 2.1$ & $3.6 \pm 1.2$ & $82.8 \pm 2.8$ & $51.3 \pm 0.6$ & $50.3 \pm 1.5$ \\
QD-G-COOH & $166.5 \pm 0.4$ & $64.5 \pm 1.1$ & $18.5 \pm 0.7$ & $101.9 \pm 1.5$ & $41.7 \pm 0.4$ & $13.4 \pm 0.7$ \\
QD-Y-COOH & $167.9 \pm 1.3$ & $69.8 \pm 0.9$ & $21.4 \pm 2.3$ & $98.1 \pm 2.2$ & $36.2 \pm 0.2$ & $14.2 \pm 0.3$ \\
QD-O-COOH & $160.3 \pm 3.6$ & $65.7 \pm 1.8$ & $21.1 \pm 0.4$ & $94.6 \pm 0.3$ & $37.4 \pm 0.1$ & $12.4 \pm 0.1$ \\
QD-R-COOH & $160.7 \pm 1.0$ & $73.5 \pm 2.5$ & $19.3 \pm 4.7$ & $87.2 \pm 3.0$ & $37.1 \pm 0.1$ & $13.2 \pm 0.5$ \\
\hline
\end{tabular}

Table S1. Contact angle and interfacial tension values of all nanofluids in addition to PAA and DI $\mathrm{H}_{2} \mathrm{O}$ in dodecane oil. 


\section{QD-G-COOH}

QD-G-PDDA

QD-G-PEG

Before
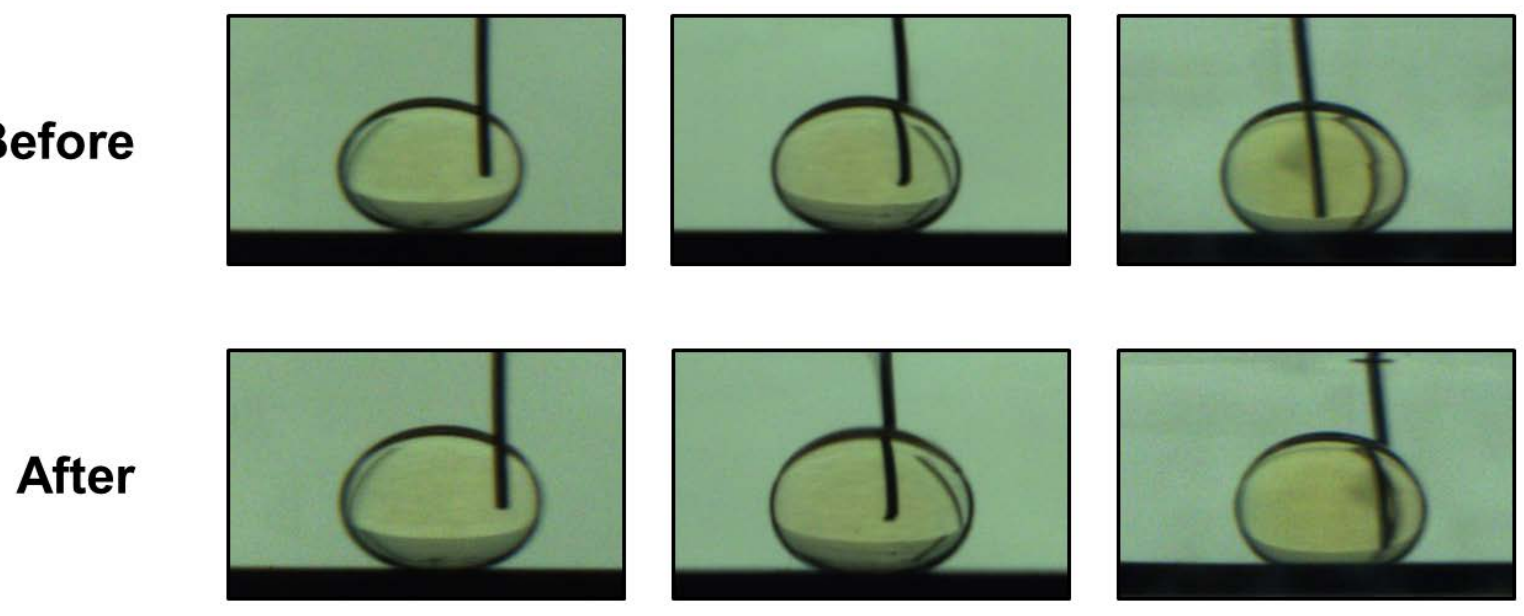

Figure S3. Representative sessile droplet images of QD-G-COOH, QD-G-PDDA, and QD-GPEG depicting contact angle hysteresis. Top: before application of voltage. Bottom: after removal of voltage once droplets have reached contact angle saturation. 


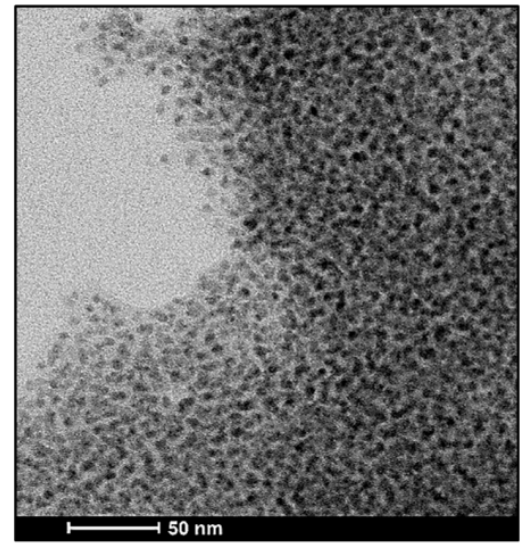

${ }^{* * *}$ diluted $3 x^{* * *}$

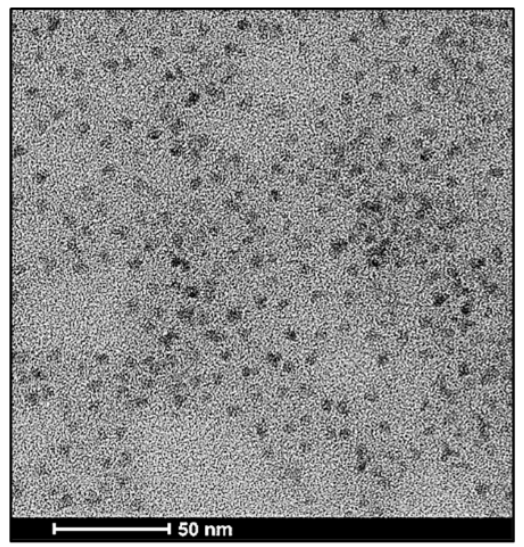

${ }^{* * *}$ diluted $40 x^{* * *}$

Figure S4. TEM images of QD-G-COOH at two dilutions depicting the effect of excess polymer. 


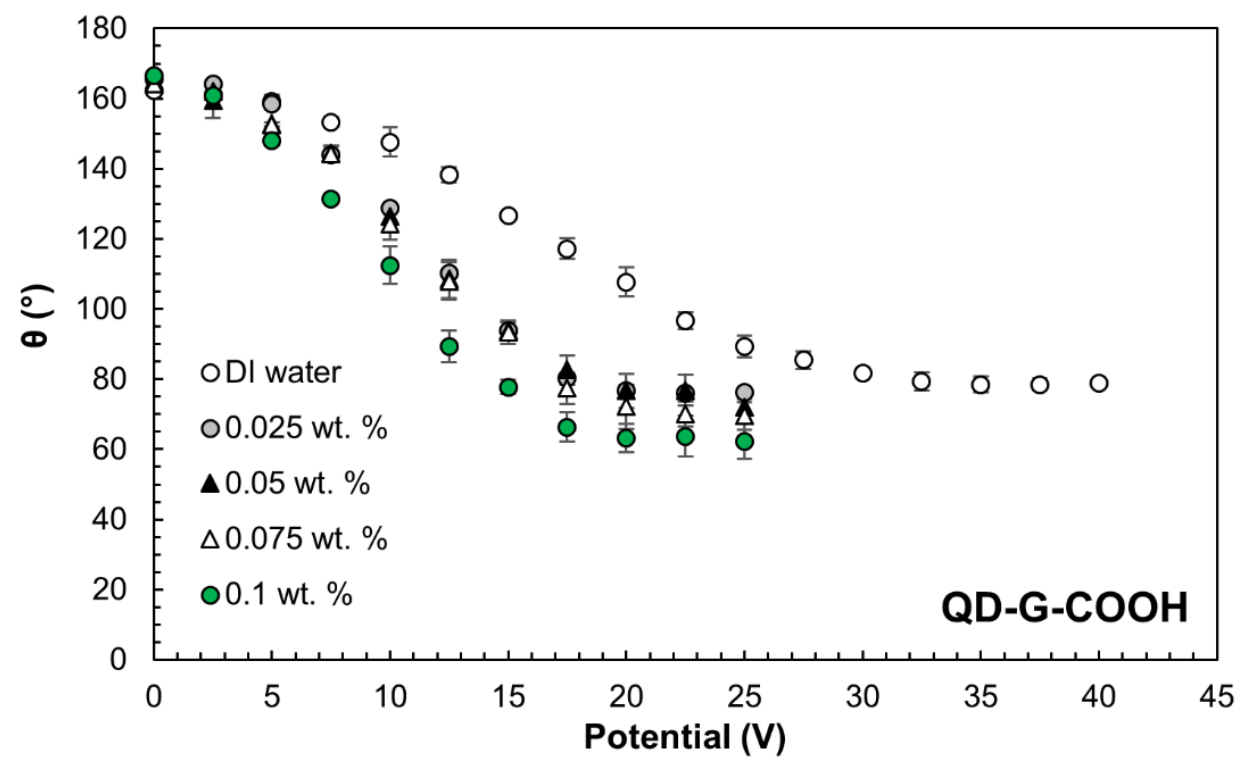

Figure S5. Contact angle change as a function of applied voltage for various concentrations of QD-G-COOH 


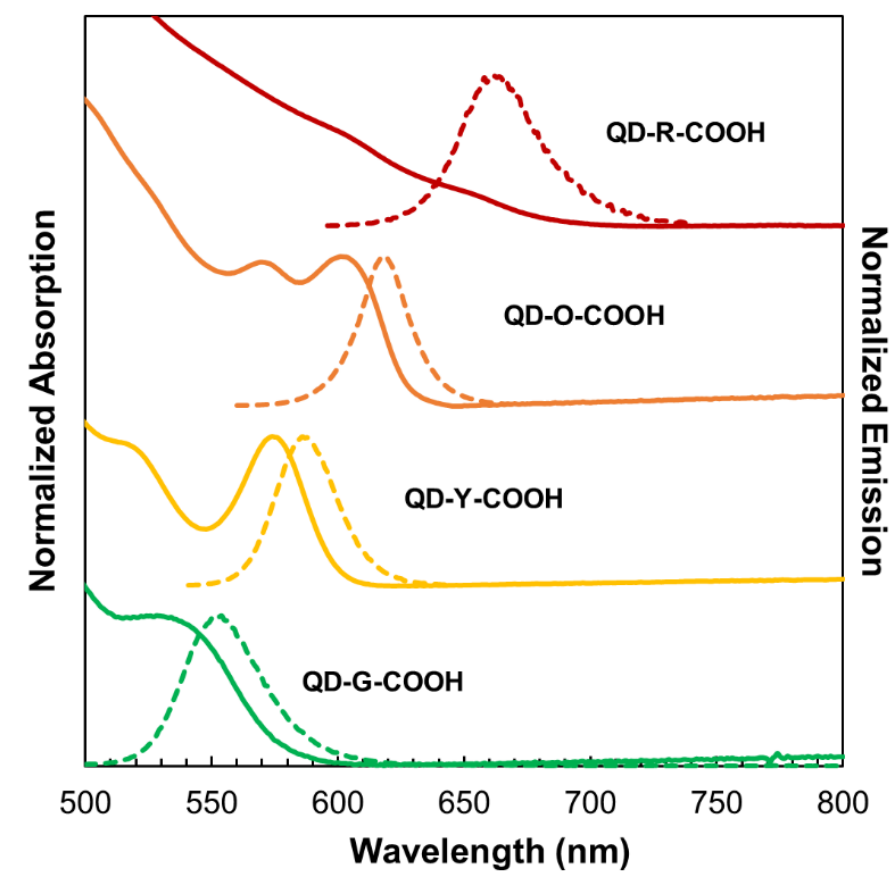

Figure S6. Normalized absorption and emission spectra of QD-G-COOH, QD-Y-COOH, QDO-COOH, and QD-R-COOH. 

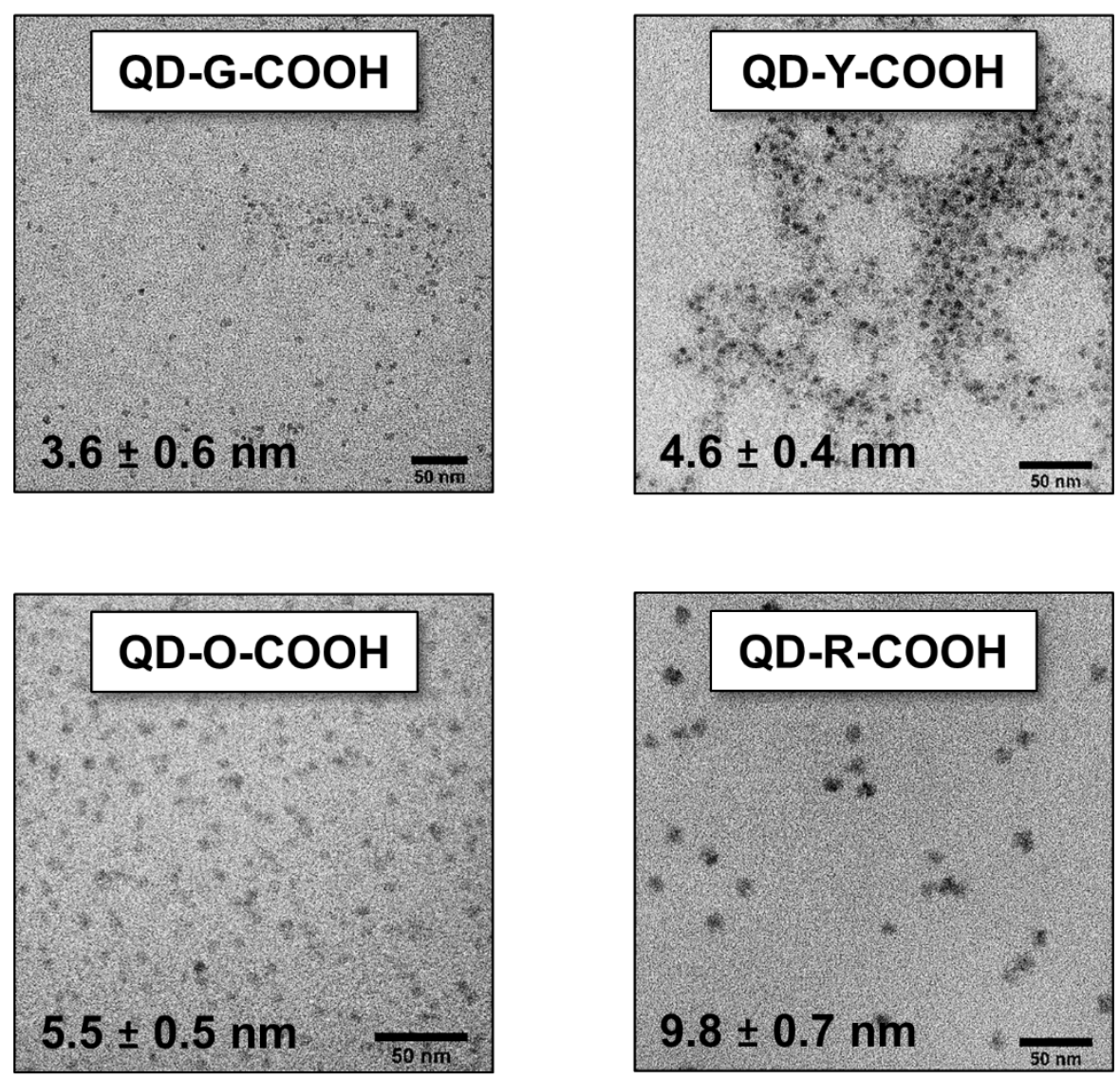

Figure S7. TEM images of QD-G-COOH, QD-Y-COOH, QD-O-COOH, and QD-R-COOH. Samples were diluted 40 times with DI water. 


\begin{tabular}{|l|lllll|}
\cline { 2 - 6 } \multicolumn{1}{c|}{} & $\theta_{\mathrm{ov}}\left(^{\circ}\right)$ & $\theta_{\text {sat }}\left(^{\circ}\right)$ & $\Delta \theta_{5 \mathrm{~V}}\left(^{\circ}\right)$ & $\Delta \theta_{\text {sat }}\left({ }^{\circ}\right)$ & $\begin{array}{l}\gamma_{L L, a p p} \\
(\mathrm{mN} / \mathrm{m})\end{array}$ \\
\hline $0.05 w t \%$ & & & & & \\
QD-G-COOH & $157.1 \pm 4.2$ & $69.4 \pm 5.7$ & $32.0 \pm 5.8$ & $92.8 \pm 3.3$ & $5.6 \pm 1.0$ \\
\hline $1.0 w t \%$ & & & & & \\
QD-G-COOH & $148.7 \pm 2.4$ & $68.3 \pm 3.1$ & $42.5 \pm 5.1$ & $80.4 \pm 4.4$ & $3.3 \pm 0.5$ \\
QD-Y-COOH & $151.7 \pm 2.0$ & $70.3 \pm 1.8$ & $46.5 \pm 6.7$ & $81.4 \pm 1.0$ & $3.2 \pm 0.4$ \\
QD-O-COOH & $150.9 \pm 1.9$ & $69.2 \pm 3.3$ & $52.9 \pm 3.7$ & $81.7 \pm 2.8$ & $2.6 \pm 0.2$ \\
QD-R-COOH & $143.9 \pm 1.7$ & $66.5 \pm 2.7$ & $56.8 \pm 0.2$ & $77.4 \pm 2.6$ & $2.0 \pm 0.1$ \\
\hline
\end{tabular}

Table S2. Contact angle and interfacial tension values of nanofluids in 0.05 wt \% and 1.0 wt \% span 80 in dodecane oil. 